\title{
Mutational analysis of the AtNUDT7 Nudix hydrolase from Arabidopsis thaliana reveals residues required for protein quarternary structure formation and activity
}

\author{
Kamil Olejnik ${ }^{1 \#}$, Danuta Płochocka², Marcin Grynberg 3 , Grażyna Goch ${ }^{4}$, \\ Wiesław I. Gruszecki ${ }^{5}$, Teresa Basińska ${ }^{6}$ and Elżbieta Kraszewska ${ }^{1 凶}$ \\ ${ }^{1}$ Department of Plant Biochemistry, ${ }^{2}$ Department of Bioinformatics, ${ }^{3}$ Department of Genetics, ${ }^{4}$ Department of \\ Biophysics, Institute of Biochemistry and Biophysics, Polish Academy of Sciences, Warszawa, Poland; ${ }^{5}$ Depart- \\ ment of Biophysics, Institute of Physics, Marie Curie-Skłodowska University, Lublin, Poland; ${ }^{6}$ Center of Mo- \\ lecular and Macromolecular Studies, Polish Academy of Sciences, Łódź, Poland
}

Received: 19 February, 2009; revised: 24 April, 2009; accepted: 12 May, 2009

available on-line: 15 May, 2009

\begin{abstract}
Arabidopsis thaliana AtNUDT7, a homodimeric Nudix hydrolase active on ADP-ribose and $\mathrm{NADH}$, exerts negative control on the major signaling complex involved in plant defense activation and programmed cell death. The structural and functional consequences of altering several amino-acid residues of the AtNUDT7 protein have been examined by site-directed mutagenesis, far-UV circular dichroism (CD), attenuated total reflection-Fourier transform infrared (ATR-FTIR) and photon correlation (PCS) spectroscopy, biochemical analysis and protein-protein interaction studies. Alanine substitutions of F73 and V168 disallowed dimer formation. Both the F73A- and V168A-mutated proteins displayed no observable enzymatic activity. Alanine substitution of the V69 residue did not significantly alter the enzyme activity and had no influence on dimer arrangement. The non-conserved V26 residue, used as a negative control, did not contribute to the enzyme quaternary structure or activity. Detailed biophysical characterization of the wild-type and mutant proteins indicates that the mutations do not considerably alter the secondary structure of the enzyme but they affect dimer assembly. In addition, mutating residues V69, F73 and V168 disrupted the binding of AtNUDT7 to the regulatory 14.3.3 protein. These are the first studies of the structure-function relationship of AtNUDT7, a Nudix hydrolase of important regulatory function.
\end{abstract}

Keywords: Arabidopsis thaliana, Nudix, AtNUDT7, mutagenesis, biophysical analysis, structure-function analysis, dimer, 14.3.3 interaction

\section{INTRODUCTION}

Nudix hydrolases are widely distributed in nature and have been identified in Archaea, Bacteria, and Eucarya. These proteins are characterized by the presence of the Nudix motif, a highly conserved amino-acid sequence; $\mathrm{GX}_{5} \mathrm{EX}_{7} \mathrm{REUXEEXGU}$ (where $U$ is usually Ile, Leu or Val). Enzymes from this family catalyze the hydrolysis of a variety of nucleoside diphosphate derivatives including NTPs, dNTPs, dinucleoside polyphosphates, nucleotide sugars, coenzyme A, NADH, NAD ${ }^{+}$and FAD. Some of these compounds play regulatory roles, whereas others are metabolic intermediates and potentially

$\triangle$ Corresponding author: Elzbieta Kraszewska, Department of Plant Biochemistry, Institute of Biochemistry and Biophysics PAS, A. Pawińskiego 5A, 02-106 Warszawa, Poland; tel.: (48) 22659 7072; fax: (48) 22 658 4636; e-mail: elzbietak@ibb. waw.pl

\#Present address: Bio-Rad Poland, Warszawa, Poland.

Abbreviations: ATR, attenuated tatal reflection; BSA, bovine serum albumine; DTT, dithiothreitol; FPLC, fast performance liquid chromatography; NBT/BCIP, S-(4-nitrobenzyl)-6-thioinosine/5-bromo-4-chloro-3-indolyl phosphate; PBS, phosphate-buffered saline; PCS, photon correlation spectroscopy; PMSF, phenylmethylsulfonyl fluoride; RMSD, root-meansquare deviation; SDS, sodium dodecyl sulphate; TBSB buffer, $10 \mathrm{mM}$ Tris/ $\mathrm{HCl}, \mathrm{pH}$ 7.5, 2\% BSA, $100 \mathrm{mM} \mathrm{NaCl}$; TBST, Tris buffer-NaCl-Tween 20. 
toxic components. Thus, the proposed role of $\mathrm{Nu}$ dix hydrolases is to control the cellular level of these compounds, which can be harmful in excess (Bessman et al., 1996).

Arabidopsis thaliana AtNUDT7, a 282 aminoacid protein encoded by the At4g12720 gene, is a homodimeric hydrolase that, in vitro, catalyzes the hydrolysis of a variety of substrates with a preference for ADP-ribose (Kraszewska et al., 2004; Olejnik \& Kraszewska, 2005) and NADH (Ogawa et al., 2005). Recent reports have shown that this hydrolase plays a very important regulatory role in plant pathogenesis and programmed cell death. Mutant A. thaliana plants, deprived of AtNUDT7, exhibit growth retardation, spontaneous cell death and increased resistance to pathogen infection (Jambunathan \& Mahalingam, 2006). Genetic analysis showed that these phenotypic changes of Atnudt7 mutants were dependent on a functional enhanced disease susceptibility 1 complex (EDS1) which controls the plant defense response and programmed cell death (Bartsch et al., 2006). It has also been suggested that AtNUDT7 is involved in maintaining redox homeostasis (Ge et al., 2007). Despite detailed examinations of the Atnudt7 mutant plants, the mode of action of this Nudix hydrolase in the cell remains unknown. Thus, the aim of this work was to gain an insight into the structure-activity correlation of the AtNUDT7 protein that could yield clues as to the regulation of its biological action. In order to elucidate the molecular mechanism of AtNUDT7 dimer formation, several conserved amino-acid residues were chosen for mutagenesis. Analyses of the constructed mutants point to the residues required for proper quarternary structure formation that is essential for the protein function.

\section{MATERIALS AND METHODS}

Chemicals. Biochemicals and alkaline phosphatase-conjugated secondary antibody were supplied by Sigma (Deisenhofen, Germany). Mouse primary anti-His-tag antibody was purchased from Invitrogen (Paisley, UK). Polyclonal anti-AtNUDT7 antibody was supplied by BioGenes (Berlin, Germany). The T87 $A$. thaliana cell line was kindly provided by Emmanuelle Jeannette from Université Pierre et Marie Curie, Physiologie Cellulaire et Moleculaire des Plantes (Paris, France). The QuikChange site-directed mutagenesis kit was purchased from Stratagene (Cedar Creek, TX, USA). The Matchmaker Yeast two-hybrid system was supplied by ClontechTakara Bio Europe (Saint-Germain-en-Laye, France). Escherichia coli BL21(DE3) Rosetta was purchased from Novagen (Darmstadt, Germany) and Saccharomyces cerevisiae PJ69-4A, a highly efficient strain for two-hybrid selection, was obtained from Dr. Philip James of the Department of Biomolecular Chemistry, University of Wisconsin (USA). cDNA encoding the 14.3.3 (GF 14 iota isoform) protein was obtained from the Arabidopsis Biological Resource Center (Ohio, OH, USA). pGEX-4T-1 vector was supplied by GE Healthcare Europe (Münich, Germany). Plasmid DNA sequencing and oligonucleotide primers used in the polymerase chain reaction were provided by the DNA Sequencing and Oligonucleotide Synthesis service at the Institute of Biochemistry and Biophysics, Polish Academy of Sciences (Warszawa, Poland).

Protein purification and enzyme assay. Expression and purification of recombinant wild-type and mutated His-tagged-AtNUDT7 proteins was performed essentially as described previously (Olejnik \& Kraszewska, 2005). cDNA encoding the 14.3.3 iota protein was cloned into pGEX-4T-1 expression vector encoding a GST tag. The recombinant GST-tagged 14.3.3 protein was expressed in E. coli BL21(DE3) Rosetta and purified as described by GE Healthcare manual. The enzymatic activity of Histagged AtNUDT7 and mutant forms was tested using ADP-ribose or NADH as substrates in a hydrolase assay. Standard reaction mixtures of $25 \mu \mathrm{l}$ contained: $50 \mathrm{mM}$ Tris/ $\mathrm{HCl} \mathrm{pH} \mathrm{7.0,5} \mathrm{mM} \mathrm{MgCl}{ }_{2}, 1 \mathrm{mM}$ DTT, $2 \mathrm{mM}$ ADP-ribose or $2 \mathrm{mM} \mathrm{NADH}$ and $0.1-1.0$ milliunits of freshly purified wt AtNUDT7, V26A or V69A forms, $0.1-50$ milliunits of the F73A or V168A mutant proteins. After incubation at $37^{\circ} \mathrm{C}$ for $30 \mathrm{~min}$ the reaction was stopped by EDTA and 1-2 units of alkaline phosphatase was added and the incubation continued for an additional $15 \mathrm{~min}$ at $37^{\circ} \mathrm{C}$. The reaction was terminated by the addition of $275 \mu$ lof $20 \mathrm{mM}$ EDTA. The resulting inorganic orthophosphate was determined colorimetrically as described earlier (Olejnik \& Kraszewska, 2005). Under optimal conditions, one unit of enzyme catalyzed the hydrolysis of $1 \mu \mathrm{mol}$ of substrate per minute.

Preparation of protein extracts from A. thaliana T87 cell culture. The plant cells were disrupted by sonication $(5 \times 30 \mathrm{~s})$ using Sonifier 250 (Branson Ultrasonic Corp., Danbury, CT, USA). Sonication was conducted in a PBS, $\mathrm{pH}$ 7.4, supplemented with $10 \mathrm{mM} \beta$-mercaptoethanol, $50 \mathrm{mM} \beta$-glycerophosphate, $100 \mu \mathrm{M} \mathrm{Na} \mathrm{VO}_{4}, 500 \mu \mathrm{M}$ PMSF, $1 \mu \mathrm{M}$ pepstatin, $1 \mu \mathrm{M}$ leupeptin and $1 \mu \mathrm{M}$ aprotinin. Following sonication the extracts were cleared by centrifugation and the supernatant was used for further studies.

Pull-down assay. For GST pull-down assays, slurry of S-hexylglutathione-agarose with the bound recombinant GST-tagged 14.3.3 iota protein was used as a bait. The resin-bound protein was incubated either with the extracts from A. thaliana T87 cells or different forms of the His-tagged AtNUDT7 
protein, for $2 \mathrm{~h}$ at $4^{\circ} \mathrm{C}$ on a rotary shaker. The resin with bound protein complexes was washed extensively with PBS supplemented with $10 \mathrm{mM} \beta$ mercaptoethanol and $0.05 \%$ Tween 20 . The protein complexes were released from the resin by boiling with SDS gel-loading buffer and the proteins were applied onto $10 \%$ SDS/polyacrylamide gels.

Western blot analysis. Western blotting was performed essentially as described in the Invitrogen manual. Proteins were separated on $10 \%$ SDS/polyacrylamide gels and transferred to nitrocellulose membrane by electroblotting. The membrane was blocked for $1 \mathrm{~h}$ at room temperature in TBSB buffer (10 mM Tris/HCl, pH 7.5, $100 \mathrm{mM} \mathrm{NaCl}$ ) containing $2 \%$ BSA and then incubated for $2 \mathrm{~h}$ in that buffer containing primary anti-His-tag antibody at a dilution of 1:2000 or polyclonal anti-AtNUDT7 antibody at a dilution of $1: 2000$. Following extensive washing in TBST $(10 \mathrm{mM}$ Tris/HCl, pH 7.5, $100 \mathrm{mM} \mathrm{NaCl}$, $0.1 \%$ Tween 20 ) to remove unbound antibody, the blots were incubated with an alkaline phosphataseconjugated secondary antibody $(1: 1000)$ in TBSB buffer for $1 \mathrm{~h}$, then washed again in TBST and developed using NBT/BCIP substrate.

Site-directed mutagenesis of recombinant AtNUDT7. Site-directed mutagenesis was performed using the QuikChange site-directed mutagenesis kit, according to the manufacturer's procedure. The plasmid pQE80L, containing the AtNUDT7 cDNA, was used as template. Four residues (V26, V69, F73 and V168) were individually mutated to alanine. Each mutant plasmid was verified by sequencing and then used to transform E. coli BL21/DE3 Rosetta. Colonies selected by ampicillin and chloramphenicol resistance were propagated for protein expression. The AtNUDT7 mutants were expressed and purified according to the procedure used for the wild-type enzyme, as described previously (Olejnik \& Kraszewska, 2005).

CD spectroscopy. Circular dichroism spectra of wild-type AtNUDT7 and its mutant forms were obtained using spectrometer an Aviv model 202. Proteins at the concentration of $4 \mu \mathrm{M}$ in $5 \mathrm{mM}$ Tris/ $\mathrm{HCl}$ buffer, $\mathrm{pH}$ 8.6, $0.5 \mathrm{mM}$ EDTA and 0.5 mM DTT were each placed in a $2 \mathrm{~mm}$-path length cuvette. Spectra were collected at $25^{\circ} \mathrm{C}$. The data were converted to molar residue ellipticity according to the equation: $[\Theta]=\theta /(10 \times \mathrm{n} \times 1 \times \mathrm{c})$, where $[\Theta]$ represents molar residue ellipticity (degree $\left.\mathrm{cm}^{2} \mathrm{dmol}^{-1}\right), \theta$ is the observed ellipticity in millidegrees, $\mathrm{n}$ is the number of amino-acid residues in the protein, 1 is the path length in $\mathrm{cm}$, and $\mathrm{c}$ is the molar protein concentration in mole/liter. Protein concentrations were determined using the spectroscopic method, with a molar absorption coefficient $\varepsilon_{280}=49100 \mathrm{M}^{-1} \mathrm{~cm}^{-1}$ calculated as described (Mach et al., 1992).

ATR-FTIR spectroscopy. Attenuated total reflection infrared (ATR-FTIR) spectra of a protein
(30 $\mu \mathrm{g})$ deposited onto ATR crystal element by means of evaporation from buffer (50 mM Hepes/ $\mathrm{KOH}, 0.5 \mathrm{mM}$ EDTA, $0.5 \mathrm{mM}$ DTT) were recorded with a Vector 33 spectrometer (Bruker, Germany). The internal reflection element was a ZnSe crystal $\left(45^{\circ}\right.$ cut) yielding ten internal reflections. Absorption spectra were recorded at a resolution of one data point every $4 \mathrm{~cm}^{-1}$, in the region between 4000 and $400 \mathrm{~cm}^{-1}$. Typically, ten scans were collected, averaged and Fourier-transformed for each measurement. As background, the buffer alone was deposited to a clean crystal, evaporated, and scanned. The instrument was continuously purged with argon for $40 \mathrm{~min}$ before and during measurements. ATR crystals were cleaned with organic solvents. All measurements were carried out at $21^{\circ} \mathrm{C}$. Spectral analysis was performed with OPUS software (Bruker, Germany).

Photon correlation spectroscopy (PCS). PCS analysis was performed at $25^{\circ} \mathrm{C}$ using a $3000 \mathrm{HSa}$ Zeta-Sizer (Malvern) apparatus. The proteins were dissolved in $50 \mathrm{mM}$ Hepes/ $\mathrm{KOH}$ supplemented by $0.5 \mathrm{mM}$ EDTA and $0.5 \mathrm{mM}$ DTT. Filtered solution containing $1 \mathrm{mg} / \mathrm{ml}$ of the AtNUDT7 protein (wt or mutants) was placed into $3 \mathrm{ml}$ cuvettes and illuminated with a laser at $633 \mathrm{~nm}$ at the scattering angle equal $90^{\circ}$. The diameters of the proteins were evaluated by taking the average of 100 measurements. The size and size distribution of the proteins were calculated using a standard CONTIN algorithm in the analysis of the PCS autocorrelation curves.

Size exclusion chromatography. Size exclusion chromatography was performed using a Superdex 20010/300 GL column and a fast performance liquid chromatography system (FPLC). The proteins $(10-20 \mu \mathrm{M})$ were applied onto column and eluted with $50 \mathrm{mM}$ Tris/HCl, pH 7.5, supplemented with $150 \mathrm{mM} \mathrm{NaCl}$, at a flow rate of $0.6 \mathrm{ml} / \mathrm{min}$.

Yeast two-hybrid analysis. Yeast two-hybrid experiments were performed according to the Clontech Matchmaker Two-Hybrid user manual. cDNAs encoding wt or mutated AtNUDT7 were subcloned into the vector pGBT9 (BD). cDNA encoding the Arabidopsis 14.3.3 protein was subcloned into the vector pGAD424 (AD). The $\mathrm{AD}$ and $\mathrm{BD}$ constructs were introduced into the yeast strain PJ69-4A by the lithium acetate method (James et al., 1996). The cotransformed clones were initially grown on plates with non-selective minimal medium lacking tryptophan and leucine $(-\operatorname{Trp} / \mathrm{Leu})$. These strains were then streaked onto selective minimal medium lacking either tryptophan, leucine and histidine (-Trp/ Leu/His) or tryptophan, leucine and adenine (-Trp/ Leu/Ade). Interaction between the AtNUDT7 forms and the 14.3.3 protein was monitored by assessing the growth of yeast co-transformants on selection media after $3-5$ days at $30^{\circ} \mathrm{C}$. 
Comparative sequence analysis and computational methodology. A protein multiple sequence alignment was constructed using homologs identified with HomoloGene (http://www.ncbi.nlm.nih. gov/entrez/query.fcgi?DB=homologene) and $A$. thaliana paralogs identified using BLAST (Altschul et al., 1990). The names of sequences used in the alignment denote the species and the GI numbers.

A search for potential structural homologs of AtNUDT7 was conducted using the MetaServer (Kelley et al., 2000; Shi et al., 2001; Lambert et al., 2002; Ginalski et al., 2003; Fischer, 2003; McGuffin \& Jones, 2003; Jaroszewski et al., 2005), HHpred (Jones, 1999; Soding, 2005) and PHYRE (Kelley et al., Web page) servers. The hypothetical three-dimensional model of the AtNUDT7 monomer was constructed using the HOMOLOGY module within the InsightII package ver. 2000.1 (Accelrys, San Diego, USA). To check the influence of particular mutations on the protein structure, the wild-type and mutated model structures were subjected to energy minimization in vacuo using the CVFF forcefield, as implemented in DISCOVER ver. 2004.1.

The ConSurf model was built using both the multiple sequence alignment and the coordinates of the AtNUDT7 model (Landau et al., 2005).

\section{RESULTS AND DISCUSSION}

\section{Mutagenesis}

Using chemical crosslinking, we previously showed that the AtNUDT7 protein exists as a dimer in solution (Olejnik \& Kraszewska, 2005). In order to elucidate the molecular mechanism of dimer formation the following mutational analysis was conducted.

To facilitate the selection of amino acids for mutagenesis, a working model of the AtNUDT7 quarternary structure was built. The AtNUDT7 hydrolase shares no significant sequence homology with Nudix hydrolases of known structure; therefore, it was analyzed using fold recognition methods. Results obtained using several servers identified the NADH pyrophosphatase from E. coli (PDB entries: 1VK6, 2GB5) as a potential structural homolog of AtNUDT7. Table I (Supplementary Materials at www.actabp.pl) explains the reasoning behind this choice of template for modeling the AtNUDT7 structure. The E. coli NADH pyrophosphatase contains a Nudix box located at the C-terminal part of the protein. The Nudix box forms a loop- $\alpha$ helix-loop structural motif which functions as a cofactor-binding and catalytic site. The motif is part of an $\alpha / \beta / \alpha$ sandwich (Nudix fold) that is commonly present in Nudix hydrolases (for review see Mildvan et al., 2005). A structure comparison performed with the help of the FATCAT server (Ye \& Godzik, 2003) showed that the C-terminal part of the AtNUDT7 hydrolase (fragment A100-E240) displays significant similarities to the C-terminal domain of the E. coli hydrolase and exhibits the Nudix fold. Analysis of the N-terminal 100 residues (M1-A100) of AtNUDT7 using the MetaServer (Ginalski et al., 2003), HHpred (Jones, 1999; Soding, 2005) and PHYRE (Kelley et al., Web page) servers indicated that this sequence should fold into $\alpha$-helices and $\beta$-sheets in a manner similar to that observed for the N-terminal domain of the bacterial enzyme. Therefore, monomer and the dimer structural models of AtNUDT7 (Figs. 1B and 2) were designed based on the structure of $E$. coli NADH pyrophosphatase.

On the basis of the sequence alignment and the working model of the protein structure, highly conserved residues of AtNUDT7 were identified (Fig. 1A and Fig. I - Supplementary Materials at www.actabp.pl). The majority of these conserved residues are present in the Nudix motif area. In the analyzed subfamily (HomoloGene orthologs and $A$. thaliana paralogs), some $\mathrm{N}$-terminal domain residues are also conserved. Based on these observations, the following residues were selected for mutagenesis: V69 located, most probably, on the surface of the N-terminal domain, in proximity to the dimer interface, F73 at the interface of the dimer in the N-terminal domain, and V168 located at the interface of the dimer in the C-terminal part of the protein (Figs. 1B and 2). The non-conserved residue V26 likely located on a side loop of the N-terminal domain, away from the dimer interface and Nudix motif, was chosen as a control since mutation of this amino acid should not result in any changes in the protein structure and activity.

Effect of the mutations on the enzymatic activity of AtNUDT7

The wild-type and mutated cDNAs were expressed in E. coli and the purified recombinant proteins were assayed to compare their kinetic parameters with ADP-ribose or NADH as the preferred substrates. The substitution of either F73 or V168 by alanine yielded enzymes with no detectable catalytic activity. Although the $K_{\mathrm{m}}$ values of $1.5 \mathrm{mM}$ for ADP-ribose and $1.4 \mathrm{mM}$ for NADH of the V69A mutant were similar to that exhibited by the wt enzyme (1.2 $\mathrm{mM}$ and $1.3 \mathrm{mM}$, respectively), the overall catalytic efficiency of V69A measured by $k_{\text {cat }} / K_{\mathrm{m}}$ was slightly lower $\left(1.3 \times 10^{3} \mathrm{M}^{-1} \mathrm{~s}^{-1}, 2.0 \times 10^{3} \mathrm{M}^{-1} \mathrm{~s}^{-1}\right)$ than that observed for the wt protein, $2.3 \times 10^{3} \mathrm{M}^{-1} \mathrm{~s}^{-1}$ and $2.7 \times 10^{3} \mathrm{M}^{-1} \mathrm{~s}^{-1}$, estimated for ADP-ribose and $\mathrm{NADH}$, respectively. As anticipated, the V26A mu- 
A

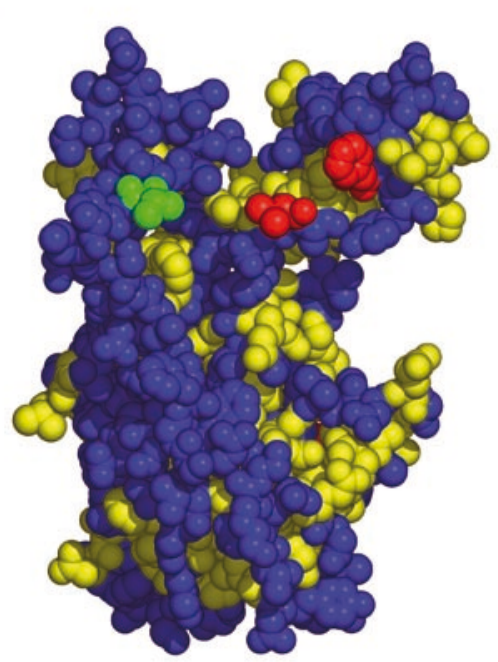

B

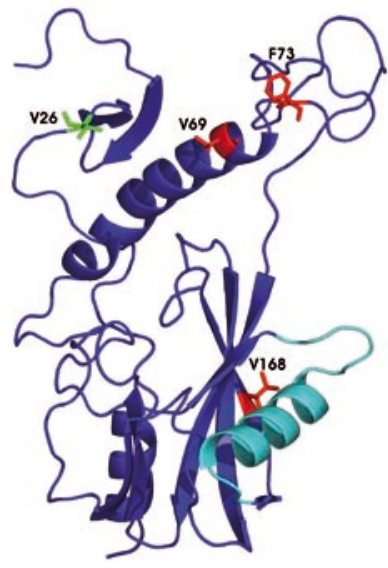

Figure 1. Model of AtNUDT7 monomer. The model was built using fold recognition method. A. Model of the protein shown as spheres. Highly conserved residues are in yellow. The mutated residues are in red (conserved) or in green (non-conserved). B. Ribbon diagram of the AtNUDT7 model with the Nudix motif highlighted in light blue. Side chains of the residues selected for study are shown as sticks and labeled according to their sequence number. The residue which has no influence on dimer formation and protein activity is shown in green, and those crucial for protein function are in red. tation did not cause any alteration in the activity compared to the wild-type (Table 1 ).

In order to detect any major changes in the backbone conformation of the mutant enzymes, which could be responsible for the altered catalytic activities of the proteins, the wild-type and mutated proteins were subjected to various biophysical analyses.

\section{Effects of the mutations on protein structure}

Circular dichroism (CD) was used to detect any major conformational changes in the secondary structure of the mutant enzymes. Figure 3 depicts CD spectra of wt and mutant AtNUDT7. The shapes of the spectra, with ellipticity minima at 208 and 222 $\mathrm{nm}$, and the $\alpha$-helical content of the mutants were similar to those of the wild-type AtNUDT7, indicating that the mutations have no or little effect on the secondary structure of the proteins (Fig. 3 and Table 2). Circular dichroism is best suited for analyzing $\alpha$-helices. Therefore, complementary FTIR spectroscopy, which is known to resolve $\beta$-sheet composition with high accuracy (for review see Calero \& Gasset, 2005), was used to study potential difference in $\beta$-type structures of the proteins. No major differences were observed in the spectral shape and $\beta$-sheet content and composition between the wildtype and the mutated proteins (Fig. 4 and Table 3). The most significant difference in total $\beta$-structures content can be observed for the V69A mutant (over $10 \%$ decrease) but it has no radical influence on the protein's activity. These data further confirm that the mutations do not introduce any major changes into the backbone conformation of the AtNUDT7 hydrolase. Thus, in order to establish how the mutations affect the quaternary structure of the protein, the wt and mutated forms of AtNUDT7 were analyzed by high performance liquid chromatography (FPLC) gel filtration. The V26A and V69A mutants were found at the same position as the dimeric $66 \mathrm{kDa}$ wild-type protein, indicating that alanine substitutions of these residues do not influence dimer formation. However, the alanine substitutions of F73 or V168 resulted in the formation of high molecular mass aggregates (Fig. 5A). In addition, Western hybridization analysis of the proteins separated by FPLC confirmed the absence of the mutated proteins at the dimer position. These data show that dimer formation is perturbed in the mutants and they also suggest that defects in dimer assembly lead to immediate aggregation of the AtNUDT7 subunits (Fig. 5B). To verify these results and to estimate the size distribution of the protein aggregates, photon correlation spectros-

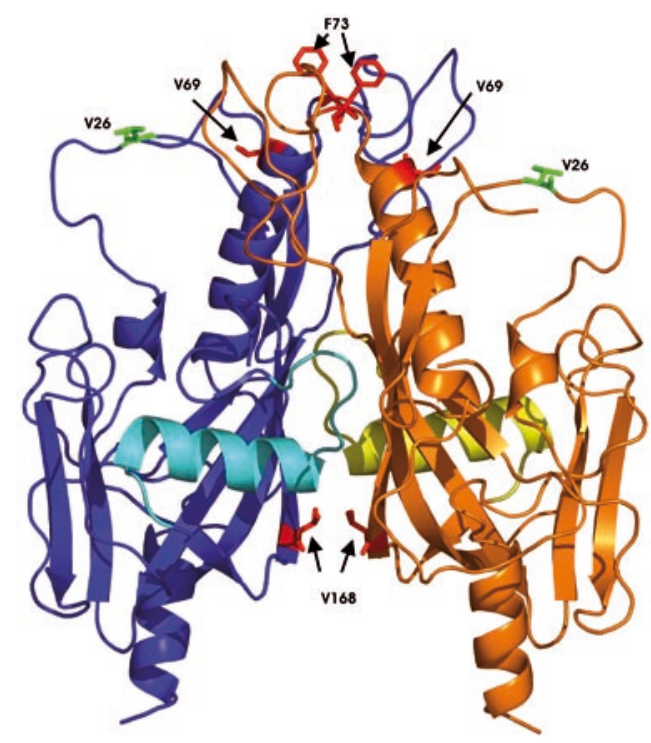

Figure 2. Model of AtNUDT7 homodimer.

One subunit is shown in blue with the Nudix motif light blue and the second monomer is colored orange with the Nudix motif yellow. Side chains of the studied residues are presented as in Fig. 1B. 
Table 1. Kinetic parameters of wt AtNUDT7 and its mutant forms.

\begin{tabular}{|c|c|c|c|c|c|c|}
\hline \multirow[t]{2}{*}{ AtNUDT7 form } & \multicolumn{2}{|l|}{$\begin{array}{l}K_{\mathrm{m}} \\
\mathrm{mM}\end{array}$} & \multicolumn{2}{|l|}{$\begin{array}{l}k_{\text {cat }} \\
\mathrm{s}^{-1}\end{array}$} & \multicolumn{2}{|l|}{$\begin{array}{l}k_{\text {cat } /} K_{\mathrm{m}} \\
\mathrm{M}^{-1} \mathrm{~s}^{-1}\end{array}$} \\
\hline & ADP-ribose & NADH & ADP-ribose & NADH & ADP-ribose & $\mathrm{NADH}$ \\
\hline wt & $1.2 \pm 0.11$ & $1.3 \pm 0.12$ & 2.7 & 3.5 & $2.3 \times 10^{3}$ & $2.7 \times 10^{3}$ \\
\hline V26A & $1.3 \pm 0.12$ & $1.3 \pm 0.12$ & 3.0 & 3.1 & $2.3 \times 10^{3}$ & $2.4 \times 10^{3}$ \\
\hline V69A & $1.5 \pm 0.12$ & $1.4 \pm 0.12$ & 2.0 & 2.8 & $1.3 \times 10^{3}$ & $2.0 \times 10^{3}$ \\
\hline F73A & ND & ND & ND & ND & ND & ND \\
\hline V168A & ND & ND & ND & ND & ND & ND \\
\hline
\end{tabular}

ADP-ribose or NADH at a concentration of 0.1 to $2 \mathrm{mM}$ were used for colorimetric measurements. The $K_{\mathrm{m}}$ and $V_{\max }$ were determined from nonlinear regression analysis. The $k_{\text {cat }}$ was calculated from $V_{\max }$ assuming one active site per monomer. One unit of enzyme catalyzes the hydrolysis of $1 \mu \mathrm{mol}$ of substrate/min under optimal conditions. ND, not determined, no observable enzymatic activity.

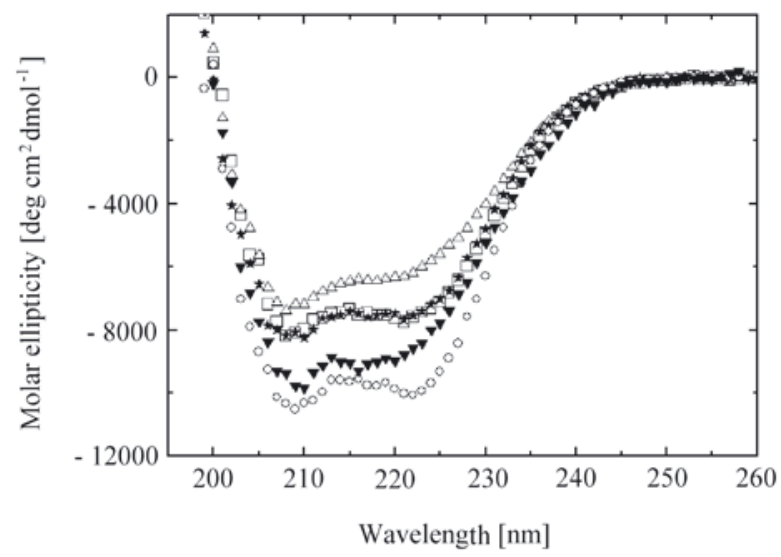

Figure 3. Circular dichroism (CD) spectra of wild-type AtNUDT7 and its mutant forms.

The spectra were obtained for wt AtNUDT7 $(\square)$ and mutants: V168A $(\Delta)$, F73A $(\boldsymbol{\nabla})$, V69A (*) and V26A $(O)$.

copy (PCS) analysis was performed. Figure 6 depicts the size and size distribution of the wt and mutated proteins. As expected, the V26A and V69A forms are similar to the wt protein, with an average size lower than $5 \mathrm{~nm}$, whereas the majority of F73A and V168A are seen as aggregates, with an average size of 16 $\mathrm{nm}$. A small amount (less than $4 \%$ ) of bigger aggre-

Table 2. Secondary structure content of wt AtNUDT7 and its mutant forms.

\begin{tabular}{lcccc}
\hline AtNUDT7 form & $\alpha$-helix & $\beta$-sheet & $\beta$-turn & Others \\
\hline \multicolumn{5}{c}{$[\%]$} \\
\hline wt & $25 \pm 1$ & $20 \pm 1$ & $17 \pm 1$ & $38 \pm 2$ \\
V26A & $31 \pm 1$ & $19 \pm 1$ & $17 \pm 1$ & $33 \pm 2$ \\
V69A & $25 \pm 1$ & $20 \pm 1$ & $17 \pm 1$ & $38 \pm 2$ \\
F73A & $28 \pm 1$ & $20 \pm 1$ & $17 \pm 1$ & $35 \pm 2$ \\
V168A & $21 \pm 2$ & $22 \pm 2$ & $18 \pm 2$ & $39 \pm 2$ \\
\hline
\end{tabular}

Circular dichroism spectra were collected as described in "Materials and Methods". Secondary structure contents were estimated using CDNN software [8].

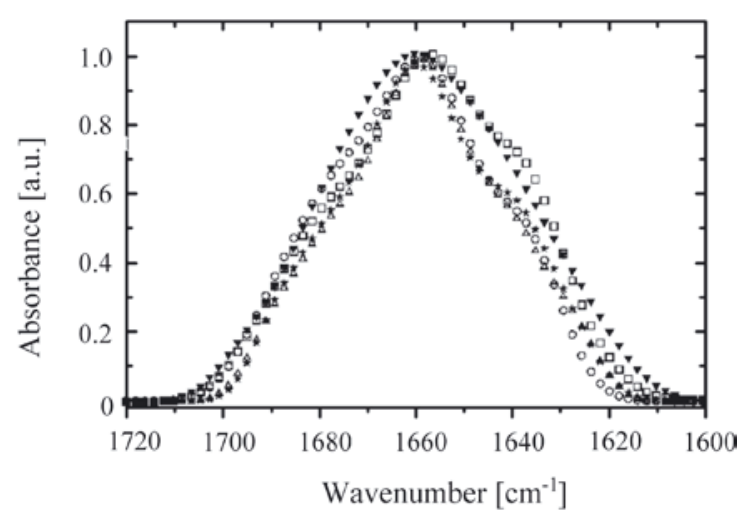

Figure 4. ATR-FTIR spectra of wild-type AtNUDT7 and its mutant forms.

The FTIR spectra were determined for wt AtNUDT7 ( $\square$ ) and for mutants: V168A $(\Delta)$, F73A $(\boldsymbol{\nabla})$, V69A (*) and V26A (O).

gates, with an average size in the range of 400-600 $\mathrm{nm}$, was also observed (not shown).

\section{Effect of the V26A, V69A, F73 and V168A mutations on binding to the 14.3 .3 protein}

The 14.3.3 proteins function as regulators or scaffold proteins in a wide range of cellular processes including those involved in signal transduction. In plants, a number of reports implicate 14.3 .3 proteins in responses to environmental, metabolic and nutritional stresses, as well as in defense responses (Roberts et al., 2002). Using the 14.3 .3 protein as a "bait" we have pulled down the AtNUDT7 hydrolase from an Arabidopsis cellular extract (Fig. 7 lane 4). This result indicates that in vivo the two proteins form a complex. To investigate the influence of the introduced mutations on the protein-protein interactions of AtNUDT7, its binding to the 14.3 .3 protein was analyzed.

Using the yeast two-hybrid system cDNAs encoding wt or mutated forms of AtNUDT7 were cloned into the BD plasmid whereas the 14.3.3 cDNA was cloned into the AD plasmid. As shown in Figs 8A and B, interactions between the 14.3.3 protein and either wild-type or V26A-mutated At- 


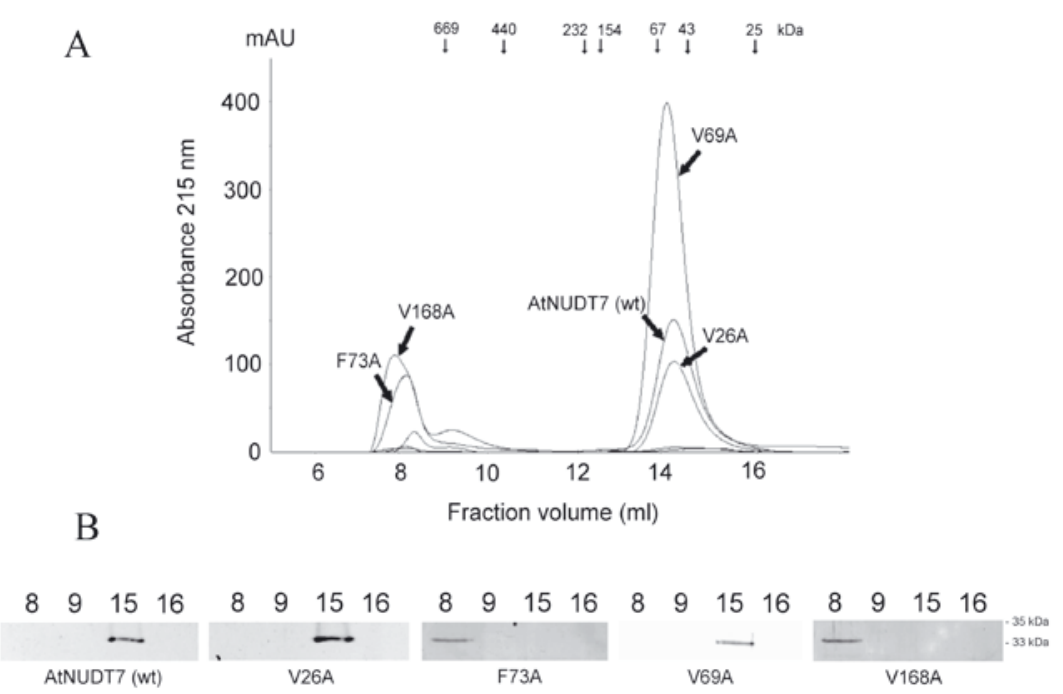

Figure 5. A. FPLC analysis of wt AtNUDT7 and its mutant forms.

Protein mass standards are indicated by arrows.

B. Western blot analysis of the recombinant proteins precipitated from the indicated FPLC fractions $(8,9,15$ and 16).

Western hybridization was performed with anti-His antibody. The positions of protein molecular mass markers are indicated on the right.

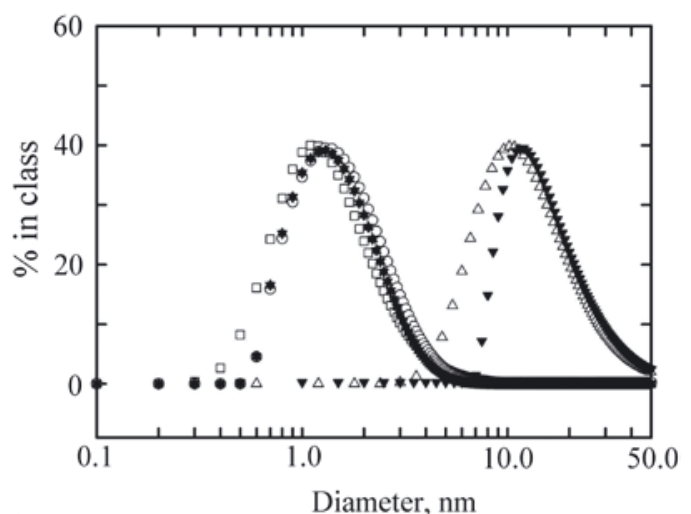

Figure 6. Photon correlation spectroscopy (PCS). PCS measurements were conducted for wt AtNUDT7 ( $\square$ ) and its mutant forms: V168A $(\Delta)$, F73A $(\boldsymbol{\nabla})$, V69A (*) and V26A (O).

NUDT7 permit growth of yeast colonies on both selective media. However, no growth was observed when the V69A, F73A or V168A mutants were tested for interactions with the 14.3 .3 protein (Fig. 8C, $\mathrm{D}$ and E), indicating that these mutated forms of AtNUDT7 are unable to bind the protein partner. The results obtained from the yeast two-hybrid experi- ment were further corroborated by pull-down and Western analyses. The 14.3.3 iota protein interacted with the wild type and the V26A mutant form of AtNUDT7 (Fig. 9 lane 3 and 4). However, no interactions between 14.3.3 and the V69A, F73A or V168A mutants were observed (Fig. 9 lane 5, 6 and 7).

The present study has identified several residues, from both the $\mathrm{N}$ - and C-terminal domains, that are crucial for quarternary structure, activity and protein-protein binding of the A. thaliana Nudix hydrolase AtNUDT7. Moreover, the investigated amino acids, although highly conserved, had not been highlighted in earlier studies on other Nudix hydrolases.

The F73 residue appears to be critical for the dimer structure. However, this residue is most likely located far away from the catalytic domain and is not involved directly in catalysis. Thus, the observed loss of enzymatic activity of this mutant results almost certainly from destabilization of the quaternary structure of the protein. Furthermore, as shown by CD and FTIR spectra, an overall conformational change of the F73A mutant protein should be minimal and could not be responsible

Table 3. ATR-FTIR spectra of wt and mutant AtNUDT7.

\begin{tabular}{|c|c|c|c|c|c|c|}
\hline $\begin{array}{l}\text { Center of the Gaus- } \\
\text { sian component band } \\
{\left[\mathrm{cm}^{-1}\right]}\end{array}$ & 1660 & 1636 & 1680 & 1694 & 1621 & 1654 \\
\hline AtNUDT7 form & $\alpha$-helix & $\beta$-sheet & $\begin{array}{l}\beta \text {-turns and } \\
\text { loops }\end{array}$ & $\begin{array}{l}\text { Antiparallel } \\
\beta \text {-sheet }\end{array}$ & $\begin{array}{l}\text { Aggregated } \\
\text { strands }\end{array}$ & Unordered \\
\hline \multicolumn{7}{|c|}{$[\%]^{*}$} \\
\hline wt & 26 & 21 & 21 & 2 & 2 & 28 \\
\hline V26A & 27 & 15 & 25 & 4 & 0 & 29 \\
\hline V69A & 29 & 19 & 15 & 3 & 3 & 31 \\
\hline F73A & 25 & 21 & 23 & 2 & 4 & 25 \\
\hline V168A & 26 & 19 & 20 & 3 & 5 & 27 \\
\hline
\end{tabular}

*S.D. of each determination does not exceed 2\%; ATR-FTIR spectra were collected as described in Materials and Methods. Secondary structure contents were estimated using Grams/AI software from ThermoGalactic (USA). 


kDa 1243
116.0 -
66.2 -
$45.0-$
$35.0-$
$25.0-$

18.4 -

Figure 7. Analysis of interactions between 14.3.3. and AtNUDT7 by pull-down assay.

Recombinant GST-14.3.3 iota was immobilized on glutathione-agarose and incubated with $A$. thaliana cell protein extracts. Empty resin and GST-bound resin incubated with A. thaliana extracts were used as controls. 1. Recombinant His-AtNUDT7 protein purified from E. coli cell extracts; 2. Empty resin; 3. GST-bound resin; 4. Recombinant GST14.3.3 iota-bound resin. Western analysis was performed using anti-AtNUDT7 antibody as described under Materials and Methods. His-AtNUDT7 purified from E. coli served as a control of Western hybridization.

for the changes in the enzymatic properties. Similarly, mutation of the V168 residue (from the C-terminal part of AtNUDT7) does not affect the protein secondary structure, but it impairs dimer formation and enzymatic activity of the protein. Both aggregating F73A and V168A forms were unable to bind the 14.3.3 protein. Protein aggregation caused by mutation of a single amino acid in superoxide dismutase- 1 is linked to familial amyotrophic lateral sclerosis, an aggressive human neuronal disease. This mutation destabilizes the dimer but the protein secondary structure is unchanged (Ray et al., 2004). It remains to be established whether the aggregating forms of AtNUDT7 would have any effect on the plant cell metabolism.
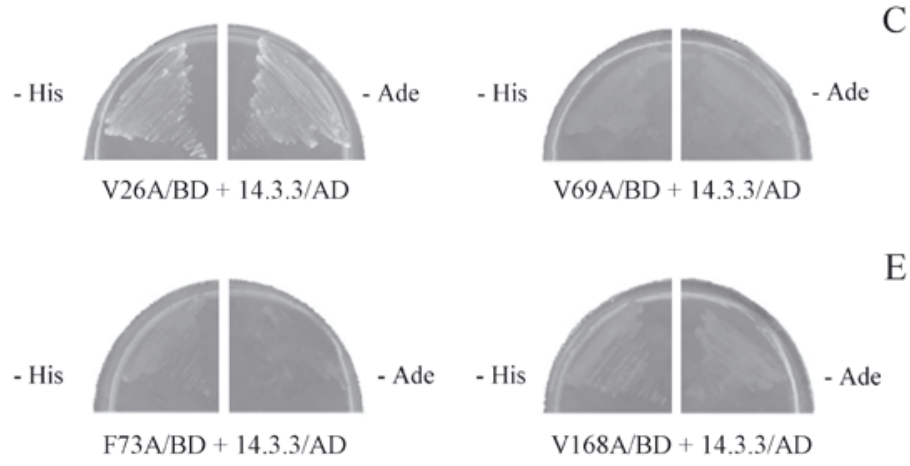

A

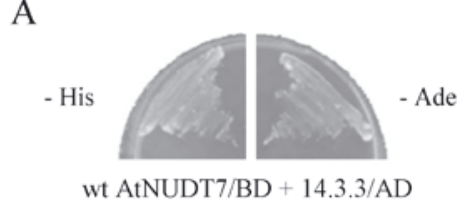

B

D

$\mathrm{F} 73 \mathrm{~A} / \mathrm{BD}+14.3 .3 / \mathrm{AD}$

$\mathrm{V} 168 \mathrm{~A} / \mathrm{BD}+14.3 .3 / \mathrm{AD}$
While in vitro the V69A mutant displays very similar physical features as the wt protein, its biochemical properties are slightly changed. The reason for this discrepancy is not clear but it may indicate that the V69A mutation introduces subtle changes in the structure of chains that are located at the dimer interface, having no influence on dimer formation but weakly affecting the catalytic efficiency of the protein. The results obtained from yeast two-hybrid and pull-down analyses indicate that the V69A mutant is unable to bind the 14.3 .3 protein. The V69A mutation has no influence on dimer assembly, therefore a failure of the V69A form to bind the 14.3.3 protein indicates that the V69 residue is directly involved in the protein-protein interaction. Recently, a mutant form of the AtNUDT7 protein was generated (Ge et al., 2007). In this mutant the conserved glutamic acid, earlier shown to be essential for catalysis conducted by Nudix enzymes (for review see Mildvan et al., 2005), was replaced by glutamine. The mutated E154Q AtNUDT7 form was unable to complement the Atnudt7 mutant phenotype, such as hyperactivation of PR genes and enhanced resistance to infection by Pseudomonas syringae. Thus, it was concluded that hydrolytic activity is required for biological function of the protein (Ge et al., 2007). However, no structural data was presented on the E154Q mutated protein. Therefore it can not be excluded that the E154Q mutation influences also other features of the AtNUDT7 protein (e.g., protein-protein binding) resulting in inability to complement the Atnudt7 mutation. It would be interesting to examine whether, its catalytically active but ineffective in the protein-protein interaction, the V69A mutant could complement the Atnudt7 phenotype.

Among the AtNUDT7 residues mutated by alanine substitution, only non-conserved V26 appears to be non-essential for protein dimerization and enzymatic activity. Most probably it is located
Figure 8. Yeast two-hybrid analysis of interactions between AtNUDT7 and 14.3.3 protein.

Interaction between the AtNUDT7 forms and the 14.3 .3 protein was monitored by assessing growth of yeast co-transformants on selection media. A. Wild type AtNUDT7 interactions; B. V26A mutant interactions; C. V69A mutant interactions; D. F73A mutant interactions; E. V168A mutant interactions. 


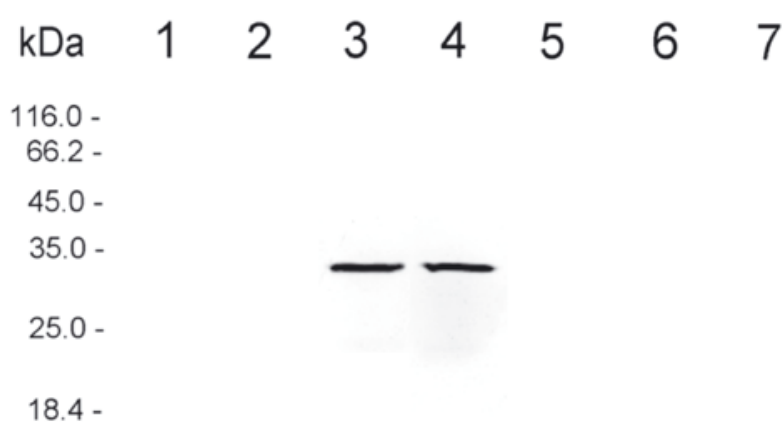

Figure 9. Analysis of interactions between wild type AtNUDT7 and its mutated forms and the $\mathbf{1 4 . 3 . 3}$ protein by pull-down assay.

Recombinant GST-14.3.3 iota or GST itself were immobilized on glutathione-agarose and incubated with recombinant wild type or mutated AtNUDT7. 1. Empty resin; 2. GST-bound resin; 3. Wild type AtNUDT7 interactions; 4. V26A interactions; 5. V69A interactions; 6. F73A; 7. V168A interactions. Western hybridization was performed with anti-His antibody as described under Materials and Methods.

neither on the dimer interface nor in the neighborhood of the active center cavity. It is well known that the residues crucial for the preservation of the higher order structure of proteins have been conserved during evolution. In the case of this hydrolase, the polypeptide structure appears to consolidate not only their enzymatic properties but also other biological functions (e.g. protein-protein interactions).

\section{Acknowledgements}

We would like to thank Dr. Philip James from the Department of Biomolecular Chemistry, University of Wisconsin for the highly efficient $S$. cerevisiae strain for two-hybrid selection, PJ69-4A. We also thank Dr. Andrzej Dziembowski from the Biology Department of the University of Warsaw, Dr. Maja Klimecka, Dr. Maja Lebska, and Arkadiusz Ciesielski, MSc., from the Department of Plant Biochemistry, IBB PAS for help with FPLC analysis.

This work was supported by the Ministry of Science and Higher Education, grants 2P06A03427 and NN303351635.

\section{REFERENCES}

Altschul SF, Gish W, Miller W, Myers EW, Lipman DJ (1990) Basic local alignment search tool. J Mol Biol 215: 403-410.

Bartsch M, Gobbato E, Bednarek P, Debey S, Schultze JL, Bautor J, Parker JE (2006) Salicylic acid-independent ENHANCED DISEASE SUSCEPTIBILITY1 signaling in Arabidopsis immunity and cell death is regulated by the monooxygenase FMO1 and the Nudix hydrolase NUDT7. Plant Cell 18: 1038-1051.

Bessman MJ, Frick DN, O'Handley SF (1996) The MutT proteins or "Nudix" hydrolases, a family of versatile, widely distributed, "housecleaning" enzymes. J Biol Chem 271: 25059-25062.

Böhm G, Mühr R, Jaenicke R (1992) Quantitative analysis of protein far UV circular dichroism spectra by neural networks. Protein Eng 5: 191-195.

Calero M, Gasset M (2005) Fourier transform infrared and circular dichroism spectroscopies for amyloid studies. Methods Mol Biol 299: 129-151.

Fischer D (2003) 3DS3 and 3DS5 3D-SHOTGUN meta-predictors in CAFASP3. Proteins 51: 434-441.

Ge X, Li G-J, Wang S-B, Zhu H, Zhu T, Wang X, Xia Y (2007) AtNUDT7, a negative regulator of basal immunity in Arabidopsis, modulates two distinct defense response pathways and is involved in maintaining redox homeostasis. Plant Physiol 145: 204-215.

Ginalski K, Elofsson A, Fischer D, Rychlewski L (2003) 3DJury: a simple approach to improve protein structure predictions. Bioinformatics 19: 1015-1018.

Gough J, Karplus K, Hughey, Chothia C (2001) Assignment of homology to genome sequences using a library of hidden Markov models that represent all proteins of known structure. J Mol Biol 313: 903-919.

Jambunathan N, Mahalingam R (2006) Analysis of Arabidopsis growth factor gene 1 (GFG1) encoding a nudix hydrolase during oxidative signaling. Planta 224: 1-11.

James P, Halladay J, Craig EA (1996) Genomic libraries and a host strain designed for highly efficient two-hybrid selection in yeast. Genetics 144: 1425-1436.

Jaroszewski L, Rychlewski L, Li Z, Li W, Godzik A (2005) FFAS03: a server for profile-profile sequence alignments. Nucleic Acids Res 33: W284-288.

Jones DT (1999) Gen THREADER: an efficient and reliable protein fold recognition method for genomic sequences. J Mol Biol 292: 195-202.

Kelley LA, MacCallum RM, Sternberg MJ (2000) Enhanced genome annotation using structural profiles in the program 3D-PSSM. J Mol Biol 299: 499-520.

Kelley L, Bennett-Lovsey R, Herber A Web Page: http:// www.sbg.bio.ic.ac.uk/phyre/

Kraszewska E, Olejnik K, Wyslouch-Cieszynska A (2004) An Arabidopsis thaliana homologue of the human GFG protein is active on ADP-ribose and FAD. Eur J Biochem 271: Supplement 71.

Lambert C, Leonard N, De Bolle X, Depiereux E (2002) ESyPred3D: Prediction of proteins 3D structures. Bioinformatics 18: 1250-1256.

Landau M, Mayrose I, Rosenberg Y, Glaser F, Martz E, Pupko T, Ben-Tal N (2005) ConSurf 2005: the projection of evolutionary conservation scores of residues on protein structures. Nucleic Acids Res 33: W299-302.

Mach H, Middaugh CR, Lewis RV (1992) Statistical determination of the average values of the extinction coefficients of tryptophan and tyrosine in native proteins. Anal Biochem 200: 74-80.

McGuffin LJ, Jones DT (2003) Improvement of the GenTHREADER method for genomic fold recognition. Bioinformatics 19: 874-881.

Mildvan AS, Xia Z, Azurmendi HF, Saraswat V, Leger PM, Massiah MA, Gabelli SB, Bianchet MA, Kang L-W, Amzel LM (2005) Structures and mechanisms of Nudix hydrolases. Arch Biochem Biophys 433: 129-143.

Ogawa T, Ueda Y, Yoshimura K, Shigeoka S (2005) Comprehensive analysis of cytosolic Nudix hydrolases in Arabidopsis thaliana. J Biol Chem 280: 25277-25283. 
Olejnik K, Kraszewska E (2005) Cloning and characterization of an Arabidopsis thaliana Nudix hydrolase. Biochim Biophys Acta 1752: 133-141.

Ray S, Nowak R, Strokovich K, Brown RH Jr, Walz T, Lansbury PT Jr ( 2004) An intersubunits disulfide bond prevents in vitro aggregation of a superoxide dismutase-1 mutant linked to familial amyotrophic lateral sclerosis. Biochemistry 43: 4899-4905.

Roberts MR, Salinas J, Collinge DB (2002) 14-3-3 proteins and the response to abiotic and biotic stress. Plant Mol Biol 50: 1031-1039.
Shi J, Blundell TL, Mizuguchi K (2001) FUGUE: sequencestructure homology recognition using environmentspecific substitution tables and structure-dependent gap penalties. J Mol Biol 310: 243-257.

Soding J (2005) Protein homology detection by HMMHMM comparison. Bioinformatics 21: 951-960.

Ye Y, Godzik A (2003) Flexible structure alignment by chaining aligned fragment pairs allowing twists. Bioinformatics 19 (Suppl 2): ii246-ii255. 